\title{
Una Mirada a la Investigación Educativa en América Latina a partir de sus Artículos
}

\section{A Look at Educational Research in Latin America from its Papers}

\author{
F. Javier Murillo* \\ Cynthia Martínez-Garrido
}

Universidad Autónoma de Madrid, España

\begin{abstract}
El presente estudio busca aportar una panorámica general de la investigación educativa desarrollada en América Latina a partir del estudio de los artículos publicados en revistas latinoamericanas de impacto y escritos por autores de la Región. Concretamente se analizan los 1.086 artículos de investigación empírica en educación escritos por autores latinoamericanos y publicados en revistas editadas en América Latina e indexadas en JCR (ISI Web of Science), Scopus o SciELO, en los años 2014, 2015 y 2016. Se revisan cuatro elementos: la autoría de los artículos, la temática de estudio, el nivel educativo que aborda y la metodología de investigación utilizada. Los resultados indican que tres países (Brasil, México y Chile) concentran el $75 \%$ de los trabajos, el 59,1\% de los autores son mujeres y el 53\% de los artículos están firmados por tres o más personas. Entre las temáticas más estudiadas destacan el Aprendizaje y desempeño escolar, los Docentes y las estrategias de Enseñanza, currículo y prácticas de enseñanza. El nivel educativo claramente más estudiado es la Educación Superior, con el 43,1\% de los artículos. Y, por último, no hay ningún enfoque metodológico prioritario, con prácticamente la mitad de artículos cualitativos, la otra mitad cuantitativos. Con toda esta información es posible concluir algunos elementos que se traducen en la necesidad de un mayor apoyo a la investigación, un replanteamiento de temas y niveles de estudio y un impulso a la investigación colaborativa.
\end{abstract}

Descriptores: Investigación; Educación; América Latina; Revistas de impacto; Calidad.

This research seeks to provide a general overview of educational research in Latin America based on the study of articles published in Latin American impact journals and written by authors of the Region. Specifically, the 1,086 articles on empirical research in education written by Latin American authors and published in journals published in Latin America and indexed in JCR (ISI Web of Science), Scopus or SciELO, during the years 2014, 2015 and 2016 are reviewed. Four elements are reviewed: the authorship of the articles, the subject of study, the level of education addressed and the research methodology used. The results indicate that three countries (Brazil, Mexico and Chile) concentrate $75 \%$ of the papers, $59.1 \%$ of the authors are women and $53 \%$ of the papers are signed by three or more people. Among the most studied topics include Learning and school performance, Teachers and Teaching strategies, curriculum and teaching practices. Higher Education is clearly the most studied, with $43.1 \%$ of the papers. And, finally, there is no priority methodological approach, with practically half of qualitative articles, the other half quantitative. With all this information, it is possible to conclude some elements that translate into the need for greater support for research, a rethinking of topics and levels of study and a boost to collaborative research.

Keywords: Research; Education; Latin America; Impact journals; Quality.

*Contacto: javier.murillo@uam.es

ISSN: 1696-4713

www.rinace.net/reice/

revistas.uam.es/reice
Recibido: $\quad 15$ de septiembre 2018

$1^{\text {a }}$ Evaluación: 1 de diciembre 2018

$2^{\text {a }}$ Evaluación: 12 de enero 2019

Aceptado: 18 de enero 2019 


\section{Introducción}

La investigación sobre productividad escolar desarrollada en países anglosajones en el pasado siglo encontró que las instalaciones y los recursos escolares no influían en el aprendizaje de los estudiantes (p. ej., Hanushek, 1997). Con la inestimable ayuda de bancos mundiales diversos, este hallazgo contribuyó a desalentar la inversión en educación en los países latinoamericanos, con terribles efectos sobre el desarrollo de millones de niños y niñas en toda la Región. Sin embargo, la investigación hecha por autores latinoamericanos, a partir del análisis de su realidad, encontró la fuerte relación entre desempeño, existencia y adecuación de los recursos escolares (Murillo y Román, 2011).

$\mathrm{El}$ anterior ejemplo solo una pequeña muestra de las demoledoras consecuencias de tomar decisiones fundamentándose en otras realidades con características radicalmente diferentes. Aunque análogas consideraciones podrían hacerse de las decisiones no basadas en un profundo conocimiento de la realidad a estudiar. De esta forma, la investigación educativa se erige como un elemento determinante para la mejora de la calidad de la educación, especialmente, en los países con mayores desafíos.

En este trabajo se busca aportar una panorámica general de la investigación educativa en América Latina a partir de uno de sus productos más relevantes: los artículos de investigación elaborados por autores latinoamericanos y publicados en revistas latinoamericanas de impacto. Así, se revisa quién investiga y qué y cómo se hace, de tal forma que se tenga una visión global de las fortalezas y lagunas de la investigación educativa en América Latina.

\section{Revisión de la literatura}

La investigación educativa, tal y como la conocemos en la actualidad, surge a finales del siglo XIX y principios del XX, cuando se aplica al campo de la Pedagogía la metodología científica-experimental (De Landsheere, 1985). Entre sus fundadores se encuentran el francés Alfred Binet (1857-1911), el suizo Édouard Claparède (1873-1940), el estadounidense Charles Judd (1873-1946), el alemán Ernst Meumann (1862-1915) de Alemania y el argentino Víctor Mercante (1862-1915).

Efectivamente, América Latina hizo una aportación decisiva de la mano de Mercante a la creación del nuevo campo científico de la investigación educativa, en los primeros años del pasado siglo. Sin embargo, hubo que esperar hasta mediados del siglo xx para que recibiera el impulso institucional definitivo, especialmente en Argentina, Brasil, Chile, México, Puerto Rico y Uruguay, y se desarrollara investigación educativa con un cierto impacto (Palamidessi, Gorostiaga y Suasnábar, 2014; Shiefelbein, 1990). Los trabajos elaborados desde los años 50 hasta los 70 , primera etapa de la investigación educativa en América Latina, se caracterizan por realizarse en un contexto de desarrollo y expansión de los sistemas educativos, y por recibir una fuerte influencia de los trabajos que se realizan en Estados Unidos y Europa fundamentalmente centrados en el diseño de medidas psicométricas, test y pruebas de rendimiento (Rivero, 1994).

Las décadas de los años 80 y 90 delimitan la segunda etapa, considerada como la de mayor auge y reconocimiento social. Está caracterizada por a) dar especial atención al contexto, tanto a nivel macro-estructural, como a las dinámicas internas de las aulas; b) adquirir un fuerte carácter explicativo promoviendo el desarrollo teórico conceptual aunque sin 
establecer líneas de investigación con prioridades claramente definidas, y c) desarrollarse en unas condiciones de escasez de investigadores estables, de recursos económicos y de acceso a publicaciones especializadas (Abraham y Rojas, 1997; Gutiérrez Serrano, 1998; Palademesi, Gorostiaga y Suasnábar, 2014).

La tercera etapa se desarrolla desde finales de los años 90 y se podría considerar que llega hasta la actualidad. Entre otros elementos, se caracteriza por la autonomía de sus organismos de investigación, el aumento de la cantidad y calidad de las publicaciones científicas y el fuerte impulso por la divulgación del conocimiento. En ese sentido es especialmente significativa la creación de diferentes directorios, repositorios y bibliotecas científicas como FAPESP-BIREME en 1992, Latindex en 1995, SciELO en 1997, RedALyC en el año 2002 o, más recientemente, la red de repositorios "LA Referencia" en 2010, con un gran impacto en la investigación (Miguel, 2011).

Durante esta última etapa se incrementa de forma considerable el número y la calidad de las revistas de investigación en la Región. Así, por ejemplo, mientras que a finales de la década de los 90 tan solo dos revistas estaban en indexadas en la base de datos Scopus (de la editorial Elsevier) -"Estudios Pedagógicos" (Chile) y "Revista Cubana de Educación Médica Superior" (Cuba)-; en el año 2000 este número se duplica, y una década después llegaba a 21 revistas. En la actualidad, un total de 36 revistas editadas en América Latina se encuentran indexadas en Scopus. Respecto a la base de datos JCR (ISI Web of Science), a finales de los años 90 no había ninguna revista latinoamericana de educación que estuviera indexada; una década más tarde, en el año 2010, tres revistas lo estaban, se trata de "Revista Latinoamericana de Investigación en Matemática Educativa-RELIME" (México) y las revistas brasileñas "Movimiento" y "Bolema. Boletim de Educacao Matematica”. En la actualidad tan sólo las dos primeras continúan indexadas en la base de datos JCR. Como parece razonable, el aumento del número de revistas ha generado que se haya incrementado el número de artículos de investigación, llegando a duplicarse en los últimos 10 años.

Pero la investigación educativa no está al margen de los avatares de la investigación científica en general. Según el Informe UNESCO sobre la Ciencia, "ningún país latinoamericano (aparte de Brasil) posee una intensidad de I+D comparable al de las economías de mercado emergentes. Para reducir esta brecha, los países deben empezar por aumentar el número de investigadores" (UNESCO, 2015, p. 216). Efectivamente, la falta de investigadores sitúa a algunos países de la Región aún muy lejos del valor promedio a nivel mundial, es decir, 1.277 investigadores por cada millón de habitantes (Palademesi, Gorostiaga y Suasnábar, 2014). Concretamente, Costa Rica es el país con mayor número de investigadores por cada millón de habitantes (1.289), seguido de Argentina (1.256) que, además, es el único país de la Región en el que se alcanza la equidad de género (el $52 \%$ de los investigadores es mujer)-. A continuación, Brasil (710), Uruguay (529) y Chile (389). México, por su parte, aunque junto con Colombia son los países con menor número de investigadores, destaca especialmente por su evolución. Según el Sistema Nacional de Investigadores mexicano, el país ha pasado de 1.300 investigadores por cada millón de habitantes a comienzos de los años 80, a más de 25.000 en la actualidad. De ellos, el $58 \%$ del área de Humanidades y Ciencias de la Conducta se dedican concretamente a la investigación educativa (Colina Escalante, 2011; UNESCO, 2015).

Volviendo a la investigación educativa, existen algunos estudios que han analizado su situación en la Región. De entre los primeros en hacerlo destacan el trabajo de García 
Huidobro y Ochoa (1978) que aborda las tendencias de la investigación educativa en América Latina, y el inventario publicado por el Centro de Investigación y Experimentación Pedagógica (1982) en el que se recogen los datos de las instituciones dedicadas a la investigación educativa en América Latina y el Caribe.

También son interesantes las revisiones de investigación realizadas para países específicos. Así, para México, destacan los estudios de Arredondo y colaboradores (1984), del COMIE (2004), de Latapí (2008), de Flores-Crespo (2009) y de Colina Escalante (2011) acerca del crecimiento de la investigación educativa y el número de investigadores en este país. En Chile pueden ser señalados los estudios de Cisternas (2011), Packer y colaboradores (2014) y Villalobos y colaboradores (2016) sobre el estado de la investigación sobre formación de profesorado, las principales revistas académicas de investigación educativa y su impacto en política educativa, o la revisión realizada por Soto Roa (2012) de los artículos publicados desde el año 1990 hasta el 2010 en las revistas chilenas de investigación educativa. Entre los trabajos que analizan el estado de la investigación educativa en Argentina destacan los estudios de Gorostiaga, Nieto y Cueli (2014) y Gorostiaga, Palamidessi y Suasnábar (2016) en los que se revisa la producción de conocimiento en las revistas del país a partir del año 2000 y la difusión de la investigación educativa.

Resulta especialmente relevante para el objetivo de esta investigación el estudio realizado por Palamidessi, Gorostiaga y Suasnábar (2014) sobre la producción de conocimiento en Argentina, Brasil, Chile, México, Paraguay y Uruguay. Los autores desarrollan un análisis de los seis países en torno crecimiento, diferenciación y especialización de prácticas, agentes e instituciones de investigación educativas, y las capacidades estatales para regular la vinculación entre agencias productoras de conocimiento y los organismos y prácticas de planificación y gobierno de cada país. Entre sus resultados destacan las fuertes diferencias con respecto a la producción de investigación educativa en los seis casos analizados, y la necesidad compartida de que las instituciones nacionales respalden el campo de la investigación educativa.

Así, aunque contamos con algunos estudios que revisan la situación de la investigación educativa en América Latina, se trata en su mayoría de estudios que presentan un análisis parcial que atañe sólo a determinados países sin lograr ofrecer una visión global de la investigación educativa en la Región. Por ello, el objetivo de la presente investigación es describir el estado actual de la investigación educativa en América Latina a partir de los artículos de investigación empírica, elaborados por autores latinoamericanos y publicados en las revistas de impacto de América Latina.

\section{Método}

Para dar respuesta a este objetivo se analizan todos los artículos publicados durante los años 2014, 2015 y 2016 en revistas de investigación educativa editadas en algún país de América Latina. Para la selección de las revistas se consideró que estuviesen indexadas en las bases de datos JCR (de Thomson Reuters), Scopus (de Elsevier) y/o SciELO (creada en colaboración entre FAPESP, BIREME y CNPq). De esta forma, se consideraron 6.601 artículos publicados en alguna de las 64 revistas con esas características: 5 de ellas son revistas JCR, 28 Scopus y 31 son revistas indexadas en la base de datos SciElo. Por países, 26 de las revistas son de Brasil (2 JRC, 16 Scopus y 8 Scielo), 13 son de México (2, 6 y 5, 
respectivamente), 5 de Chile (1, 2 y 3), 5 de Colombia (2 Scopus y 2 SciElo), 4 de Venezuela (1 Scopus y 3 Scielo), 3 de Argentina, 2 de Costa Rica, 2 de Perú y 2 de Uruguay, todas ellas en SciElo y, por último, Cuba con 1 publicación en la base Scopus. Ningún otro país de América Latina tiene revistas de investigación educativa "de impacto".

De cada revista revisada se escogieron los artículos de investigación empírica en educación (se descartaron ensayos, revisiones de investigación, editoriales, cartas al editor, noticias, reseñas y notas informativas) y se seleccionaron sólo aquellos artículos donde al menos uno de los firmantes indica que su filiación profesional se ubica en América Latina. Con ello, de los ya mencionados 6.601 artículos publicados en las revistas analizadas a lo largo de los tres años del estudio, se seleccionaron 1.086 (un 16,5\%). De ellos, 291 se publicaron en 2014 (26,8\%), 352 en 2015 (32,4\%), y 443 en 2016 (40,8\%), con una distribución muy desigual entre países (figura 1).

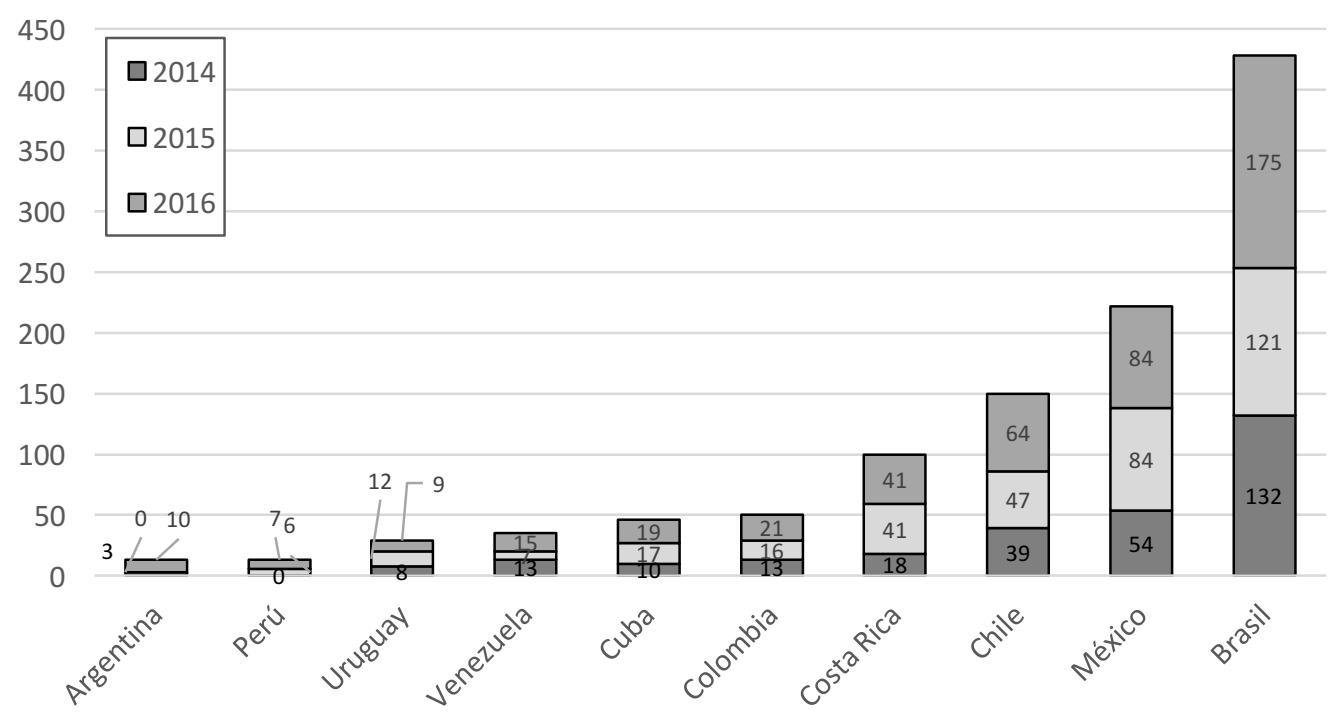

Figura 1. Número de artículos analizados por año en cada país de edición de la revista Fuente: Elaboración propia.

La presente investigación aborda el estudio de cuatro grandes temáticas:

1. Autoría, con tres variables de estudio: Número de personas firmantes, País de trabajo de cada una de ellas y su ponderación por artículo; y Género de cada una de las personas firmantes.

2. Temas de estudio: Métodos de enseñanza y currículo; Evaluación educativa; Abandono escolar; Gestión, liderazgo y organización; Docentes; Sistemas y políticas educativas; Familias; Validación de instrumentos; Libros de texto; TICTecnologías de la Información y la Comunicación; Desarrollo cognitivo; Desarrollo socio-afectivo; Educación rural e intercultural; Producción científica; Bullying; Educación inclusiva, y Preparación para el trabajo (cuadro 1).

3. Nivel educativo que aborda: Educación Infantil, Educación Primaria, Educación Secundaria, Formación Profesional, Educación Superior, Educación de Adultos, Formación Permanente, varios niveles, y genérico.

4. Método de investigación: Investigación experimental, Ex post facto descriptivo, Ex post facto explicativo, Investigación etnográfica, Investigación biográfico- 
narrativa, Estudio de caso cualitativo, Análisis documental, Método mixto, Investigación cualitativa con entrevistas, Investigación histórica, Investigación evaluativa, Investigación fenomenológica, Investigación-Acción, Investigación cualitativa con grupos de discusión, Investigación observacional y Teoría fundamentada.

Cuadro 1. Descripción de las temáticas de estudio

\begin{tabular}{|c|c|}
\hline TEMA & DESCRIPCIÓN \\
\hline Métodos de enseñanza & Estrategias de enseñanza, currículo y prácticas de enseñanza. \\
\hline Evaluación educativa & $\begin{array}{l}\text { Evaluación de estudiantes, programas o instituciones, estrategias } \\
\text { de evaluación y sistemas de acreditación de programas. }\end{array}$ \\
\hline Abandono escolar & Abandono escolar, repetición de curso y fracaso escolar. \\
\hline $\begin{array}{l}\text { Liderazgo y } \\
\text { organización escolar }\end{array}$ & $\begin{array}{l}\text { Gestión de la escuela, dirección escolar y organización de centros } \\
\text { educativos. }\end{array}$ \\
\hline Docentes & $\begin{array}{l}\text { Formación docente inicial y permanente, características, identidad, } \\
\text { creencias y conocimientos, empleo docente, competencias, } \\
\text { necesidades y evaluación del desempeño docente. }\end{array}$ \\
\hline $\begin{array}{l}\text { Sistemas y políticas } \\
\text { educativas }\end{array}$ & $\begin{array}{l}\text { Calidad de la educación, equidad, acceso a la educación, reforma } \\
\text { escolar, segregación escolar, legislación educativa y políticas } \\
\text { educativas públicas. }\end{array}$ \\
\hline Familias & $\begin{array}{l}\text { Relación de la familia con los estudiantes y su educación, relación } \\
\text { familia-escuela y maltrato familiar. }\end{array}$ \\
\hline $\begin{array}{l}\text { Validación de } \\
\text { instrumentos }\end{array}$ & Construcción y validación de instrumentos, escalas o índices. \\
\hline Libros de texto & $\begin{array}{l}\text { Libros de texto, materiales didácticos escritos o manuales escolares } \\
\text { o universitarios. }\end{array}$ \\
\hline TIC & $\begin{array}{l}\text { Desarrollo y uso de las tecnologías de la información y la } \\
\text { comunicación en la escuela y sus efectos. }\end{array}$ \\
\hline Desarrollo cognitivo & Aprendizaje y desempeño escolar de materias académicas. \\
\hline Desarrollo socio-afectivo & $\begin{array}{l}\text { Desarrollo socio-afectivo de los estudiantes tal como actitudes, } \\
\text { valores, habilidades sociales, creatividad, motivaciones, } \\
\text { autoestima, bienestar o desarrollo de la sexualidad. }\end{array}$ \\
\hline $\begin{array}{l}\text { Educación rural e } \\
\text { intercultural }\end{array}$ & $\begin{array}{l}\text { Educación intercultural e indígena, experiencias en contextos } \\
\text { étnicos y culturales minoritarios y educación rural. }\end{array}$ \\
\hline Producción científica & Estudios sobre producción académica o científica sobre educación. \\
\hline Bullying & $\begin{array}{l}\text { Descripción, causas y consecuencias del maltrato escolar entre } \\
\text { pares. }\end{array}$ \\
\hline Educación inclusiva & $\begin{array}{l}\text { Inclusión, Educación especial, barreras al estudiante o discapacidad } \\
\text { física o psíquica. }\end{array}$ \\
\hline $\begin{array}{l}\text { Preparación para el } \\
\text { trabajo }\end{array}$ & $\begin{array}{l}\text { Relación escuela-trabajo, preparación laboral y perfiles } \\
\text { vocacionales, entre otros. }\end{array}$ \\
\hline
\end{tabular}

Fuente: Elaboración propia.

\section{Resultados}

Como se ha señalado, en los años 2014, 2015 y 2016 se publicaron 1.086 artículos de investigación empírica en educación en revistas de América Latina de impacto firmados por, al menos, una persona que trabaja en la Región. A continuación, se hace una revisión de los mismos atendiendo a cuatro elementos: los autores y autoras, los temas de estudio, los niveles educativos que abordan y el método de investigación empleado. 


\subsection{Las autorías}

Conocer las características de los autores y autoras de los artículos aporta una interesante visión de las características de la investigación en América Latina. Así, el análisis del número firmantes da cuenta de la colaboración en la investigación educativa, el lugar donde trabajan da información sobre el peso de cada país en la investigación y, por último, el estudio del género de las personas que firman aporta datos de su distribución en la Región.

De los 1.086 artículos, 205 son firmados por una sola persona (el 18,9\%), 409 por dos personas (el $37,7 \%$ ), por tres personas 285 (el 26,2\%), por cuatro $133(12,2 \%)$, por cinco $41(3,8 \%)$ y por seis 12 (el 1,2\%). No es fácil valorar estas cifras por sí solas, así, se podría decir que la colaboración es escasa dado que más de la mitad $(56,5 \%)$ es firmado solo por uno o dos investigadores, aunque no es menos cierto que el $81,1 \%$ de los artículos se firman entre varias personas. En todo caso, se verifica que la colaboración entre numerosos investigadores, propio de las ciencias experimentales, no se da en Educación.

El segundo análisis es profundizar en el país en el que trabajan las personas firmantes. Así, estimando el peso a partir de las aportaciones ponderadas de cada firmante, el país que, con diferencia, más ha publicado en estos años es Brasil, que concentra el 35,9\% de todo lo publicado. Le siguen a distancia México y Chile, con un $19,1 \%$ y $18,7 \%$, y ya más lejos Colombia (6,4\%), Argentina (5,3\%), Costa Rica (4,8\%) y Cuba (2,9\%). Tras ellos, una serie de países con una producción muy baja (inferior a 20 artículos): Venezuela $(1,8 \%)$, Uruguay $(1,5 \%)$, Perú $(0,9 \%)$ y Ecuador $(0,3 \%)$. Desde Honduras y Nicaragua solo se han publicado 2 artículos, uno desde República Dominicana y Puerto Rico, y medio artículo desde Panamá. Hay que destacar especialmente que en estos tres años analizados no se ha publicado ningún artículo empírico de educación en revistas de América Latina firmado por investigadores que trabajan en Bolivia, El Salvador, Guatemala y Paraguay (figura 2).

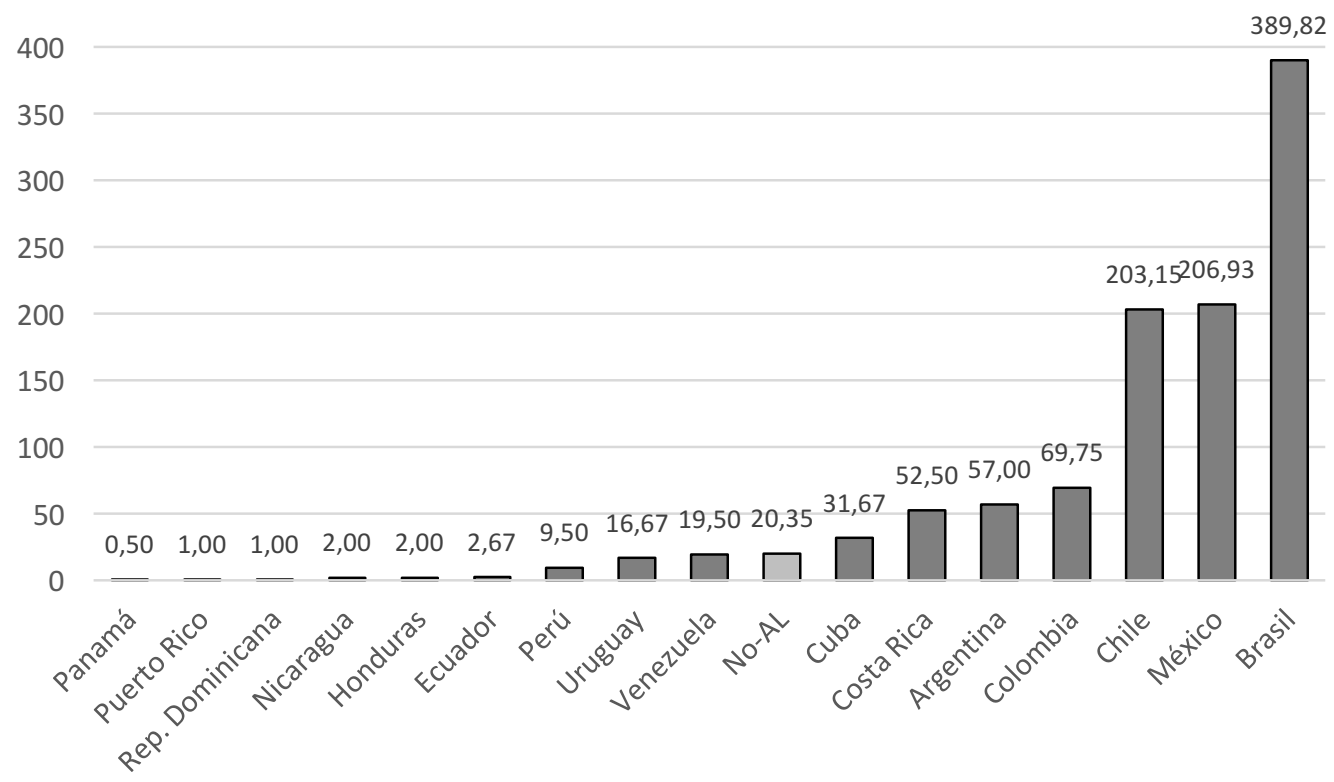

Figura 2. Distribución de los artículos de investigación educativa en América Latina por país de trabajo del/la autor/a

Nota: Datos ponderados en función del número de autores/as.

Fuente: Elaboración propia. 
Otro dato llamativo es que firman 64 autores o autoras que no trabajan en América Latina acompañando a latinoamericanos. Su peso ponderado es de 20,35 artículos lo que supone apenas un 1,9\% del total, y refleja la escasa colaboración que se da con personas fuera de la Región. Esos 64 investigadores son mayoritariamente españoles (38, un 59,4\% del total de autores del exterior), seguidos de portugueses (12, un 18,8\%) y estadounidenses (8, el $12,5 \%)$.

Una mirada más profunda la aporta el análisis de la posición de los diferentes autores en los artículos. En el cuadro 2 se muestra toda la información. Una primera idea que se extrae de la misma es que si se compara el país de trabajo del primer firmante y el peso ponderado de cada país, las diferencias son mínimas, lo que indica que el análisis por país solo del primer autor es una buena aproximación. Las mínimas diferencias las aportarían los firmantes que trabajan fuera de América latina, que solo en once casos son los primeros en aparecer en la lista de autores.

Cuadro 2. Número de autores/as y su posición en cada país

\begin{tabular}{|c|c|c|c|c|c|c|c|c|}
\hline & \multirow{2}{*}{$\begin{array}{c}1^{\mathrm{ER}} \\
\text { AUTOR } \\
\end{array}$} & \multirow{2}{*}{$\begin{array}{c}\mathbf{2}^{\mathbf{o}} \\
\text { AUTOR } \\
\end{array}$} & \multirow{2}{*}{$\begin{array}{c}\mathbf{3}^{\mathrm{ER}} \\
\text { AUTOR } \\
\end{array}$} & \multirow{2}{*}{$\begin{array}{c}4^{\circ} \\
\text { AUTOR } \\
\end{array}$} & \multirow{2}{*}{$\begin{array}{c}5^{\mathrm{a}} \\
\text { AUTOR } \\
\end{array}$} & \multirow{2}{*}{$\begin{array}{c}6^{\circ} \\
\text { AUTOR } \\
\end{array}$} & \multicolumn{2}{|c|}{ Ponderado } \\
\hline & & & & & & & Total & $\%$ \\
\hline Argentina & 57 & 37 & 17 & 5 & 2 & - & 57,00 & 5,25 \\
\hline Brasil & 394 & 336 & 148 & 62 & 14 & 6 & 389,82 & 35,90 \\
\hline Chile & 204 & 172 & 95 & 43 & 21 & 3 & 203,15 & 18,71 \\
\hline Colombia & 73 & 54 & 39 & 9 & 2 & - & 69,75 & 6,42 \\
\hline Costa Rica & 52 & 34 & 16 & 6 & 1 & - & 52,50 & 4,83 \\
\hline Cuba & 31 & 30 & 26 & 13 & 8 & 3 & 31,67 & 2,92 \\
\hline Ecuador & 3 & 2 & 2 & - & - & - & 2,67 & 0,25 \\
\hline Honduras & 2 & - & - & - & - & - & 2,00 & 0,18 \\
\hline México & 208 & 158 & 96 & 36 & 3 & 1 & 206,93 & 19,05 \\
\hline Nicaragua & 2 & 1 & 1 & - & - & - & 2,00 & 0,18 \\
\hline Panamá & O & 1 & $\mathrm{O}$ & 1 & - & - & 0,50 & 0,05 \\
\hline Perú & 10 & 6 & 3 & 1 & 1 & & 9,50 & 0,87 \\
\hline Puerto Rico & 1 & - & - & - & - & - & 1,00 & 0,09 \\
\hline Rep. Dominicana & 1 & 1 & 1 & - & - & - & 1,00 & 0,09 \\
\hline Uruguay & 17 & 11 & 2 & - & - & - & 16,67 & 1,53 \\
\hline Venezuela & 20 & 13 & 8 & 3 & - & - & 19,50 & 1,80 \\
\hline No-AL & 11 & 25 & 18 & 8 & 2 & - & 20,35 & 1,87 \\
\hline
\end{tabular}

Fuente: Elaboración propia.

Resulta revelador relacionar el país donde se publica el artículo (figura 1) con el de trabajo de los autores (figura 2). De entrada, y como parece razonable, existe una estrecha relación entre ambos elementos: los países que tienen más revistas y, por tanto, publican más artículos de investigación educativa también son los países con mayor producción. Sin embargo, se dan algunos interesantes desajustes que merecen la pena ser destacados. Y quizá el más llamativo es que hay países que producen mucho más de lo que publican y otros que publican más de lo que producen. Entre los primeros destaca Argentina que publica 4 veces más de lo que produce (67 y 13), pero también Chile (203 y 15) y Colombia (70 y 59), a los que hay que añadir, lógicamente, los países sin revistas de impacto. Por el contrario, países que producen menos que lo que publican son: Costa Rica (52 y 100), Venezuela (19 y 35), Uruguay (17 y 29), Cuba (31 y 46), Brasil (389 y 428) y México (206 y 222).

Sin embargo, esa mirada es quizá muy limitada. Es posible profundizar más contrastando el país de publicación y en el que trabaja el primer autor (cuadro 3). Así se encuentra que: 
- En Brasil, y en menor medida en Costa Rica y Cuba, los investigadores eligen en su mayoría su propio país para investigar. En los tres casos más del 75\% de los artículos están publicados en el país donde trabaja el primer autor. Concretamente, en Brasil esto ocurre con el 94,2\% de los artículos; en Costa Roca esa cifra es del 80,8\%, y en Cuba el 77,4\%. Sin embargo, las razones de por qué esto ocurre son diferentes. En Brasil la razón fundamental es el idioma, en Costa Rica es la existencia de una gran oferta para poder publicar que disuade de la internacionalización de los artículos, y en Cuba quizá la dificultad de publicar fuera del país.

- En Uruguay, Venezuela, México y Perú también la mayoría de los artículos se publican en revistas del país donde trabaja el primer autor, pero esto ocurre en menor medida que en los países antes señalados. En Uruguay es del 70,6\% de los artículos, en Venezuela el 70,0\%, en México el 67,8\% y en Perú el 60,0\%.

- En Chile aproximadamente la mitad de los artículos cuyo primer autor es chileno se publican en otro país, especialmente en México (14,2\%) y Brasil (13,2\%).

- Y, por último, en Colombia y, especialmente, en Argentina es más frecuente publicar fuera que dentro, lo que les hace países con una fuerte internacionalización de su producción. Los autores argentinos publican, en primer lugar, en México (un 29,9\% de los artículos), después en Brasil (un 21,1\%) y solo en tercer lugar en la propia Argentina (un 14,0\%). En el caso de Colombia, aunque uno de cada tres artículos se publica en el propio país, el 19,2\% lo hace en Chile y el 16,4\% en México.

Cuadro 3. Distribución de artículos de investigación educativa en América Latina en función del país donde se edita la revista, para cada país del primer firmante

\begin{tabular}{|c|c|c|c|c|c|c|c|c|c|c|}
\hline \multirow[b]{2}{*}{$\begin{array}{l}\text { PAÍ́S DEL } \\
\text { PRIMER } \\
\text { FIRMANTE }\end{array}$} & \multicolumn{10}{|c|}{ PAÍS DONDE SE EDITA LA REVISTA } \\
\hline & 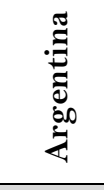 & 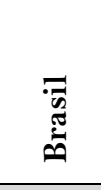 & $\stackrel{\mathscr{Z}}{\overparen{Z}}$ & $\frac{\frac{\pi}{0}}{\frac{0}{0}}$ & نَّ & $\frac{\pi}{\Xi}$ & 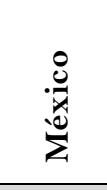 & טים & 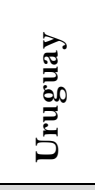 & $\begin{array}{l}\frac{\pi}{d} \\
\frac{\pi}{N} \\
\frac{d}{0} \\
>\end{array}$ \\
\hline Argentina & 14,04 & 21,05 & 12,28 & 8,77 & 7,02 & - & 29,82 & - & 5,26 & 1,75 \\
\hline Brasil & 0,25 & 94,16 & 1,52 & 0,76 & 0,51 & 0,25 & 2,28 & - & 0,25 & - \\
\hline Chile & 0,98 & 13,24 & 48,53 & 4,90 & 9,80 & $4,4+1$ & 14,22 & 1,47 & 0,98 & 1,47 \\
\hline Colombia & 1,37 & 5,48 & 19,18 & 32,88 & 5,48 & 2,74 & 16,44 & - & 2,74 & 13,70 \\
\hline Costa Rica & 1,92 & 1,92 & 1,92 & - & 80,77 & 3,85 & 3,85 & 1,92 & 3,85 & - \\
\hline Cuba & - & 3,23 & 3,23 & 6,45 & 3,23 & 77,42 & 6,45 & - & - & - \\
\hline Ecuador & - & 33,33 & - & - & - & - & 33,33 & - & - & 33,33 \\
\hline Honduras & - & - & - & - & 100,00 & - & - & - & - & - \\
\hline México & - & 1,92 & 10,10 & 2,40 & 8,65 & 2,40 & 67,79 & 1,44 & 2,88 & 2,40 \\
\hline Nicaragua & - & - & - & - & 50,00 & - & 50,00 & - & - & - \\
\hline Panamá & - & - & - & - & - & - & - & - & - & - \\
\hline Perú & - & - & - & 10,00 & - & 20,00 & 10,00 & 60,00 & - & - \\
\hline Puerto Rico & - & - & - & - & - & - & 100,00 & - & - & - \\
\hline Rep. Dominicana & - & - & - & - & - & 100,00 & - & - & - & - \\
\hline Uruguay & - & 17,65 & - & - & - & - & 11,76 & - & 70,59 & - \\
\hline Venezuela & - & 5,00 & - & - & 20,00 & - & - & - & 5,00 & 70,00 \\
\hline No AL & $\mathrm{O}$ & 3 & 1 & $\mathrm{O}$ & 2 & $\mathrm{O}$ & 4 & $\mathrm{O}$ & $\mathrm{O}$ & 1 \\
\hline
\end{tabular}

Fuente: Elaboración propia. 
Lógicamente los países que no tienen ninguna revista de impacto, sus escasas publicaciones son en otro país. Es el caso de investigadores de Ecuador, Honduras, República Dominicana, Nicaragua, Panamá y Puerto Rico.

La última mirada referida a las personas que firman los artículos se centra en su género. Y los datos son claros. La investigación educativa en América Latina está liderada por mujeres. Efectivamente, los 1.086 artículos están firmados por 2.693 personas, de las cuales el 1.592 son mujeres, lo que representa el 59,1\% del total.

Si se analiza en función de la posición de la firma, los datos no sufren apenas alteraciones: el 59,4\% de las personas que firman en primer lugar son mujeres, el 59,8\% de los que lo hacen en segundo lugar, el 59,3\% de los que lo hacen en tercer, el 58,3\% de los que lo hacen en cuarto, el 63,0\% de los que lo hacen en quinto y, por último, en los 13 artículos con seis firmantes seis son mujeres, lo que significa un $46,2 \%$.

Esa distribución, sin embargo, es diferente en función del país de procedencia (figura 3). Analizando exclusivamente la persona que firma en primer lugar, se observa que, salvo Nicaragua (con apenas dos artículos), en todos los países al menos la mitad de los firmantes son mujeres. El porcentaje es muy alto en Argentina, Cuba y Uruguay, los tres superando el $70 \%$ de investigadoras $(79,0 \%, 74,2 \%$ y $70,6 \%$ respectivamente). Entre el $60 \%$ y el $70 \%$ de mujeres en Ecuador (66,7\%), Venezuela $(65,0 \%)$ y Brasil $(64,2 \%)$; y es inferior a la media en Perú (50,0\%), Colombia (50,7\%), Chile (51,5\%), México (52,9\%) y Costa Rica (57,8\%). Se necesitarían estudios posteriores para relacionar esta producción con el número de académicas en cada país.

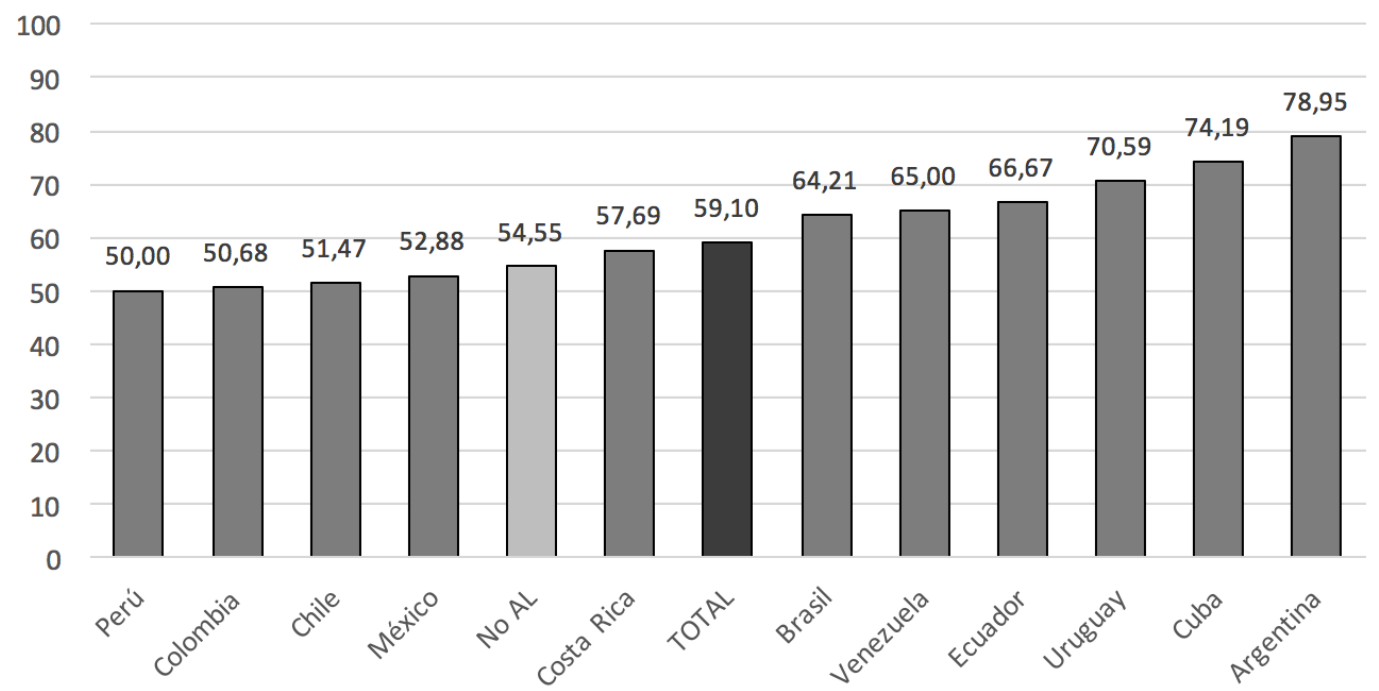

Figura 3. Porcentaje de mujeres autoras en los artículos de investigación educativa en

América Latina, en cada país

Fuente: Elaboración propia.

\subsection{Temáticas de estudio}

Como no puede ser de otra forma, la investigación educativa desarrollada en alguno de los países de América Latina aborda una amplia variedad de temáticas (figura 4). Entre ellas destacan: 
- Aprendizaje y desempeño escolar de materias académicas, especialmente Lectura, Matemáticas y Ciencias. En estos tres años analizados se han publicado 134 artículos sobre esta temática, lo que supone el 12,3\% de la producción sobre investigación educativa.

- Docentes. Es la segunda temática más abordada por la investigación latinoamericana con 129 artículos, lo que supone el 11,9\% del total. Bajo este epígrafe se encuentran trabajos sobre Formación docente inicial y permanente, características, identidad, creencias y conocimientos, empleo docente, competencias, necesidades y evaluación del desempeño docente.

- Estrategias de enseñanza, currículo y prácticas de enseñanza. En estos tres años analizados se han elaborado sobre esta temática 113 artículos que suponen el 10,4\% de los trabajos, lo que le sitúa en el tercer puesto de interés.

- Educación inclusiva, educación especial, barreras al estudiante o discapacidad física o psíquica. Son 109 estudios, que suponen el 10,0\% del total, le ubican en cuarto lugar.

- Sistema educativo y políticas educativas sobre calidad de la educación, equidad, acceso a la educación, reforma escolar, segregación escolar, legislación educativa, etc. Con menos del centenar de artículos publicados se encuentran los trabajos que tienen una mirada conjunta sobre el sistema educativo y sus políticas, en concreto 93 textos, lo que supone un $8,6 \%$ del total.

- Desarrollo socio-afectivo tal como actitudes, valores, habilidades sociales, creatividad, motivaciones, autoestima, bienestar o desarrollo de la sexualidad. Supone la sexta temática más abordada, es decir, el 8,2\% de la productividad.

- Desarrollo y uso de las tecnologías de la información y la comunicación en la escuela y sus efectos. Más alejado, con 69 trabajos (lo que significa un 6,4\%).

- Construcción y validación de instrumentos. Son 54 artículos, es decir, un 5,0\% del total.

- Gestión de la escuela, dirección escolar y organización de centros educativos. Son 48 artículos los significa un $4,4 \%$ del total.

- Educación intercultural e indígena, experiencias en contextos étnicos y culturales minoritarios y educación rural. Con apenas 43 (4,0\%) investigaciones.

Y, a partir de aquí, temáticas que suponen menos del 4\% del total: abandono escolar, repetición de curso y fracaso escolar $(3,4 \%)$, relación escuela-trabajo, preparación laboral y perfiles vocacionales $(3,3 \%)$, relación de la familia con los estudiantes y su educación, relación familia-escuela y maltrato familiar $(3,0 \%)$, evaluación de estudiantes, programas o instituciones, estrategias de evaluación y sistemas de acreditación de programas (2,7\%), descripción, causas y consecuencias del maltrato escolar entre pares $(2,1 \%)$, estudios sobre producción académica o científica sobre educación $(1,8 \%)$ y, por último, investigaciones sobre libros de texto, materiales didácticos escritos o manuales escolares o universitarios $(1,8 \%)$. 


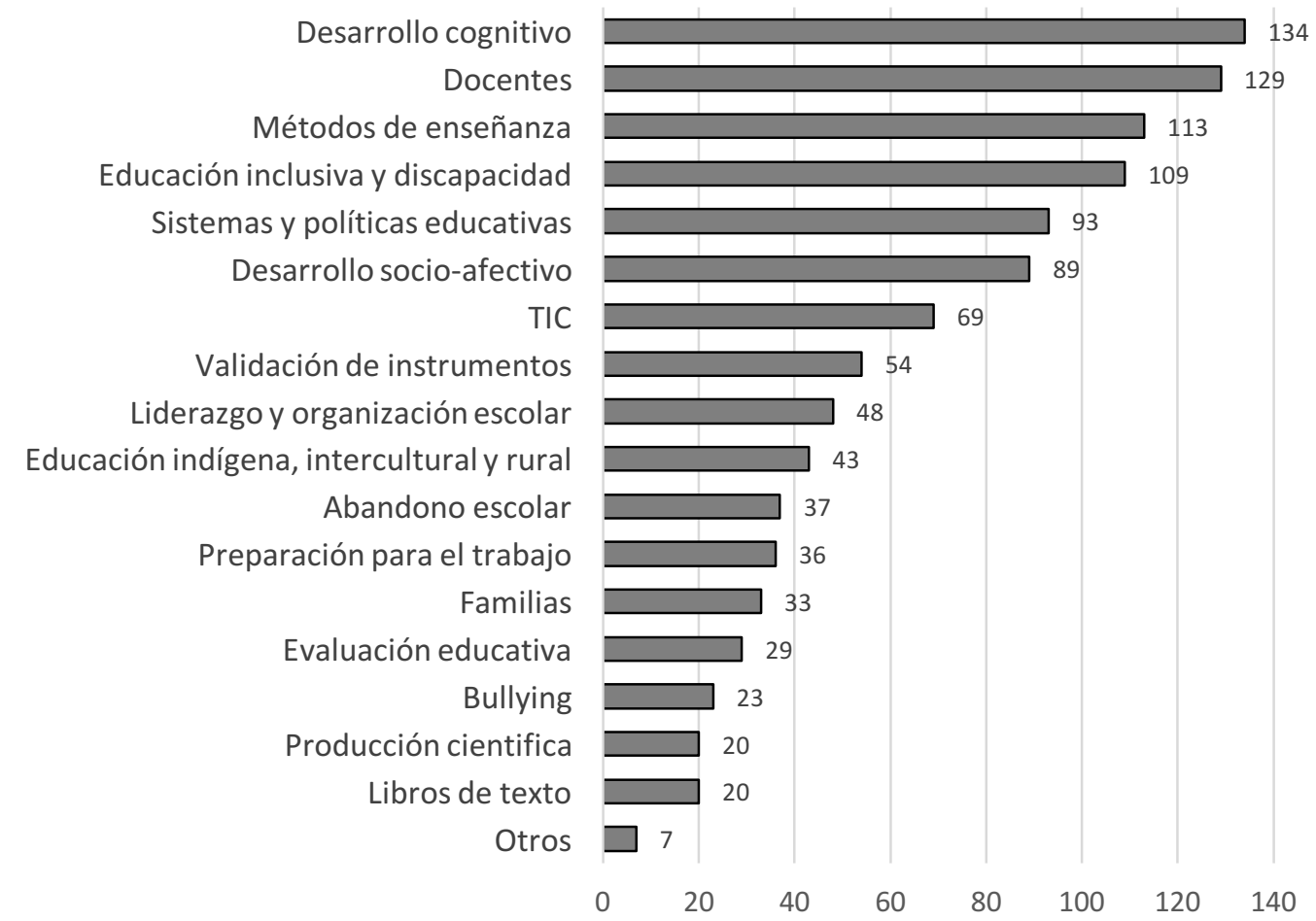

Figura 4. Distribución de los artículos de investigación educativa en América Latina en función del tema de estudio Fuente: Elaboración propia.

Una visión más detallada puede obtenerse analizando cómo esas temáticas se distribuyen entre los diferentes países, con lo que se detectan los intereses de los investigadores, así como las tradiciones de los países (cuadro 4). Profundizando exclusivamente en los seis países con más de 50 artículos es posible encontrar estos resultados:

- Argentina, en primer lugar, tiene una distribución de temas muy parecida al conjunto de América Latina. Destaca el trabajo realizado sobre Desarrollo cognitivo de los estudiantes (17,5\% de su producción) y sobre Familias (5,3\%), pero también la ausencia de investigaciones sobre muchas de las temáticas, como Liderazgo y organización escolar.

- Brasil, el país con una mayor investigación de la Región, con uno de cada tres de los artículos publicados en América Latina, sobresale por su interés en Educación inclusiva, discapacidad y educación especial, con un 16,5\% de los trabajos sobre esa temática.

- Chile, el tercer país de América Latina con mayor producción, destaca por sus trabajos sobre Liderazgo y organización escolar (un 11,3\%) y sobre Desarrollo cognitivo (un $15,2 \%$ ).

- Colombia, por su parte, tiene una temática muy marcada relacionada con el estudio de las Tecnologías de la información y la comunicación en educación, con un $26,0 \%$ de sus artículos. 
- Costa Rica, por su parte, destaca en términos relativos, con su preocupación por los Métodos de enseñanza, por los trabajos de Producción científica y de Abandono escolar.

- México, el segundo país con mayor producción, aborda todas las temáticas y en una proporción muy parecida a la del promedio de la Región. Por destacar algo tiene una importante tradición en Educación indígena, intercultural y rural, en Construcción y validación de instrumentos o en estudios que relacionan el Trabajo y la escuela.

Cuadro 4. Distribución de artículos de investigación educativa en América Latina en función del tema abordado, por país de los autores

\begin{tabular}{|c|c|c|c|c|c|c|c|c|c|c|c|}
\hline & 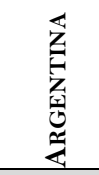 & 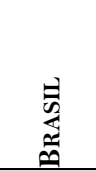 & 視 & \begin{tabular}{l}
$\mathbb{4}$ \\
\multirow{3}{*}{} \\
0 \\
0 \\
0
\end{tabular} & 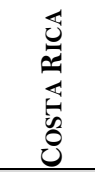 & 它 & 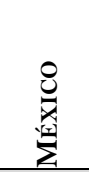 & 量 & 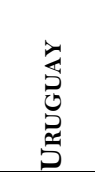 & 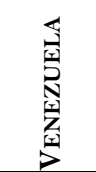 & $\underset{6}{2}$ \\
\hline $\begin{array}{l}\text { Desarrollo cognitivo } \\
\text { del estudiante }\end{array}$ & 17,54 & 10,66 & 15,20 & 8,22 & 5,77 & 16,13 & 14,90 & - & 11,76 & 10,00 & 12,34 \\
\hline Docentes & 15,79 & 12,44 & 12,75 & 6,85 & 11,54 & 9,68 & 10,58 & 30,00 & 11,76 & 5,00 & 11,88 \\
\hline Método de enseñanza & 14,04 & 10,91 & 8,33 & 8,22 & 17,31 & 25,81 & 7,69 & 10,00 & 5,88 & 10,00 & 10,41 \\
\hline $\begin{array}{l}\text { Educación inclusiva, } \\
\text { discapacidad }\end{array}$ & - & 16,50 & 8,82 & 8,22 & 11,54 & - & 6,25 & - & 5,88 & - & 10,04 \\
\hline $\begin{array}{l}\text { Sistemas educativos, } \\
\text { políticas }\end{array}$ & 12,28 & 8,12 & 9,31 & 9,59 & 7,69 & 9,68 & 7,21 & - & 17,65 & 5,00 & 8,56 \\
\hline $\begin{array}{l}\text { Desarrollo socio- } \\
\text { afectivo }\end{array}$ & 12,28 & 6,35 & 7,35 & 8,22 & 9,62 & 9,68 & 8,65 & 20,00 & 11,76 & 15,00 & 8,20 \\
\hline TIC & 8,77 & 3,81 & 4,41 & 26,03 & 3,85 & 3,23 & 4,33 & 10,00 & 17,65 & 15,00 & 6,35 \\
\hline $\begin{array}{l}\text { Validación de } \\
\text { instrumentos }\end{array}$ & - & 3,30 & 5,39 & 2,74 & 5,77 & 6,45 & 8,65 & 10,00 & - & 10,00 & 4,97 \\
\hline $\begin{array}{l}\text { Liderazgo y } \\
\text { organización esc. }\end{array}$ & - & 3,05 & 11,27 & 4,11 & 1,92 & - & 2,40 & - & 5,88 & 15,00 & 4,42 \\
\hline $\begin{array}{l}\text { Educación } \\
\text { intercultural y rural }\end{array}$ & - & 3,55 & 4,41 & 4,11 & 3,85 & - & 6,73 & 10,00 & 0,00 & 0,00 & 3,96 \\
\hline Abandono escolar & 5,26 & 3,05 & 2,94 & 5,48 & 7,69 & 0,00 & 2,88 & 10,00 & - & - & 3,41 \\
\hline $\begin{array}{l}\text { Preparación para el } \\
\text { trabajo }\end{array}$ & 1,75 & 3,05 & 2,94 & - & 1,92 & 6,45 & 6,25 & - & - & 5,00 & 3,31 \\
\hline Familias & 5,26 & 3,05 & 1,96 & 2,74 & 3,85 & - & 4,33 & - & - & 5,00 & 3,04 \\
\hline Evaluación educativa & - & 3,55 & 1,96 & 2,74 & - & - & 3,37 & - & 5,88 & - & 2,67 \\
\hline Bullying & 1,75 & 2,28 & 0,49 & 1,37 & 1,92 & - & 3,85 & - & 5,88 & - & 2,12 \\
\hline Libros de texto & 1,75 & 3,81 & 0,49 & - & - & - & 0,48 & - & - & 5,00 & 1,84 \\
\hline Producción científica & 3,51 & 1,52 & 1,96 & - & 5,77 & 9,68 & 0,96 & - & - & - & 1,84 \\
\hline Otros & - & 1,02 & - & 1,37 & 0,00 & 3,23 & 0,48 & - & - & - & 0,64 \\
\hline
\end{tabular}

Fuente: Elaboración propia.

\subsection{Nivel educativo de estudio}

La investigación educativa en América Latina está especialmente centrada en el estudio de la Educación Superior. Efectivamente, de los 1.086 artículos analizados, 468 analizan ese nivel educativo, lo que supone un $43,1 \%$ del total de la producción (figura 5). A mucha distancia le siguen las investigaciones sobre alguna temática referida a Educación Primaria (20,6\%) y a Educación Secundaria (18,1\%). Llama la atención el poco interés que hay por la Educación Infantil (solo 35 investigaciones se centran en este nivel, el 3,2\% de la producción), así como Educación de personas adultas (1,7\%) o Formación Profesional $(0,5 \%)$. 


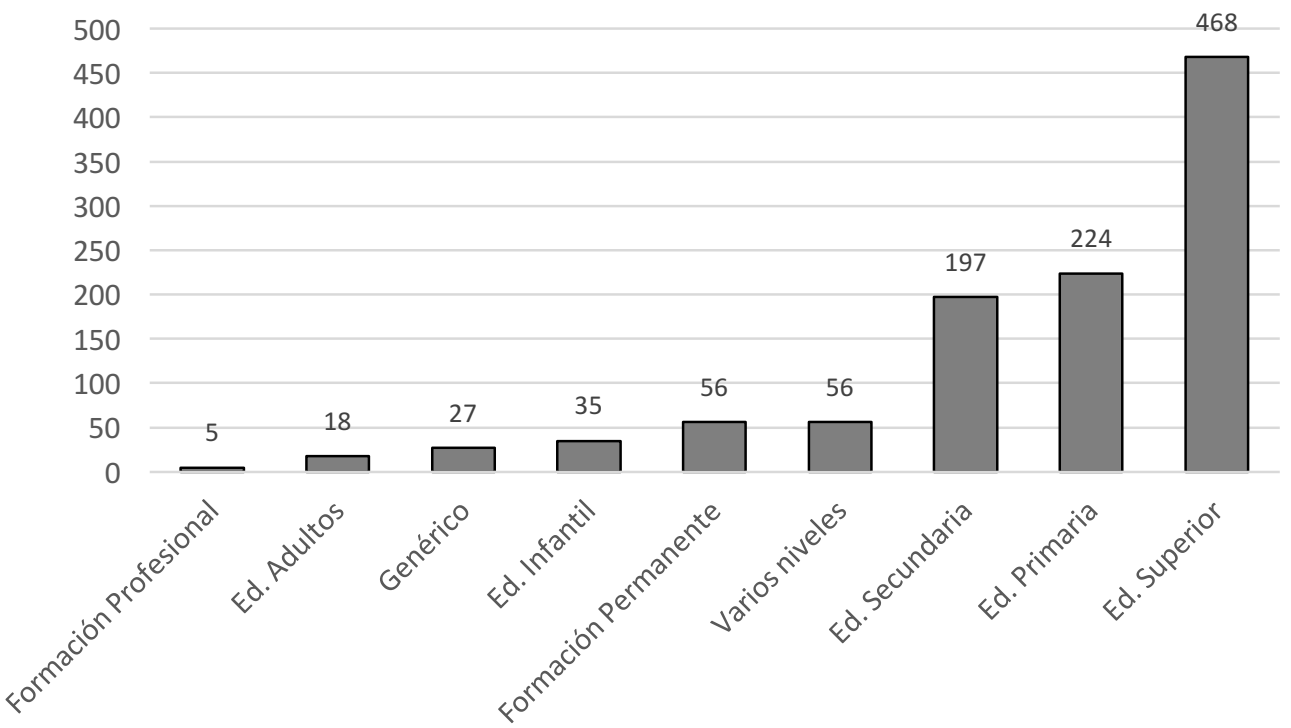

Figura 5. Distribución de los artículos de investigación educativa en América Latina en función del nivel educativo de estudio

Fuente: Elaboración propia.

De nuevo una mirada por países aporta interesantes resultados acerca de las tradiciones o intereses de investigación de los mismos (cuadro 5). Los países con una menor producción (sin revistas de impacto) ofrecen los valores porcentuales más extremos. Así, llaman poderosamente la atención dos países Perú y Uruguay. Perú, en primer lugar, porque prácticamente concentra toda su producción en el estudio de la Educación Superior (el $90 \%)$, y en Uruguay está focalizado en Educación Secundaria (64,7\%) siendo el país de la Región con menos producción relativa sobre Educación Superior y Educación Primaria.

Cuadro 5. Distribución de artículos de investigación educativa en América Latina en función del nivel educativo tratado, por país de los autores

\begin{tabular}{|c|c|c|c|c|c|c|c|c|c|c|c|}
\hline & 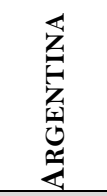 & 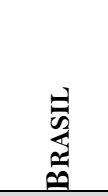 & 鼠 & $\begin{array}{l}\overleftarrow{x} \\
\sum_{0}^{0} \\
0 \\
0 \\
0 \\
0\end{array}$ & 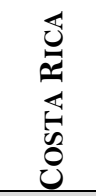 & 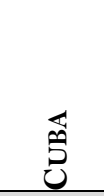 & 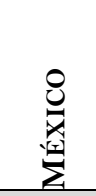 & 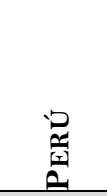 & 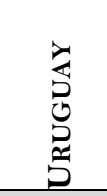 & 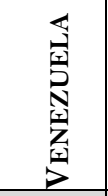 & 於 \\
\hline Ed. Superior & 42,11 & 32,49 & 39,22 & 56,16 & 51,92 & 67,74 & 54,33 & 90,00 & 29,41 & 50,00 & 43,09 \\
\hline Ed. Primaria & 17,54 & 25,63 & 22,06 & 17,81 & 19,23 & 6,45 & 15,87 & - & 5,88 & 25,00 & 20,63 \\
\hline Ed. Secundaria & 22,81 & 17,26 & 17,16 & 15,07 & 13,46 & 3,23 & 20,19 & 10,00 & 64,71 & 20,00 & 18,14 \\
\hline $\begin{array}{l}\text { Formación } \\
\text { Permanente }\end{array}$ & - & 7,87 & 3,92 & 2,74 & 3,85 & 12,90 & 3,85 & - & - & - & 5,16 \\
\hline Varios niveles & 7,02 & 5,58 & 9,31 & 4,11 & 1,92 & 3,23 & 2,88 & - & - & - & 5,16 \\
\hline Ed. Infantil & 5,26 & 3,55 & 3,92 & 2,74 & 1,92 & - & 2,40 & - & - & 5,00 & 3,22 \\
\hline Genérico & 3,51 & 3,81 & 1,47 & 1,37 & 5,77 & 6,45 & 0,48 & - & - & - & 2,49 \\
\hline Ed. Adultos & 1,75 & 3,05 & 1,96 & - & 1,92 & - & - & - & - & - & 1,66 \\
\hline $\begin{array}{l}\text { Formación } \\
\text { Profesional }\end{array}$ & - & 0,76 & 0,98 & - & - & - & - & - & - & - & 0,46 \\
\hline
\end{tabular}

Fuente: Elaboración propia. 


\subsection{Método de investigación utilizado}

Como no puede ser de otra forma, existe una amplia variedad de métodos de investigación utilizados en los diferentes artículos (figura 6). Así, en primer lugar, aunque la distinción entre investigación "cualitativa" y "cuantitativa" se torna ambigua y caduca, es posible afirmar que, aproximadamente, la mitad de la investigación educativa de carácter empírica que se elabora y publica en América Latina es de carácter cualitativo y la otra mitad cuantitativo. Los estudios que declaran usar un método "mixto" suponen apenas un 5,9\% del total. Resulta importante destacar que, en la actualidad, ningún enfoque metodológico tiene prioridad sobre el otro en América Latina.

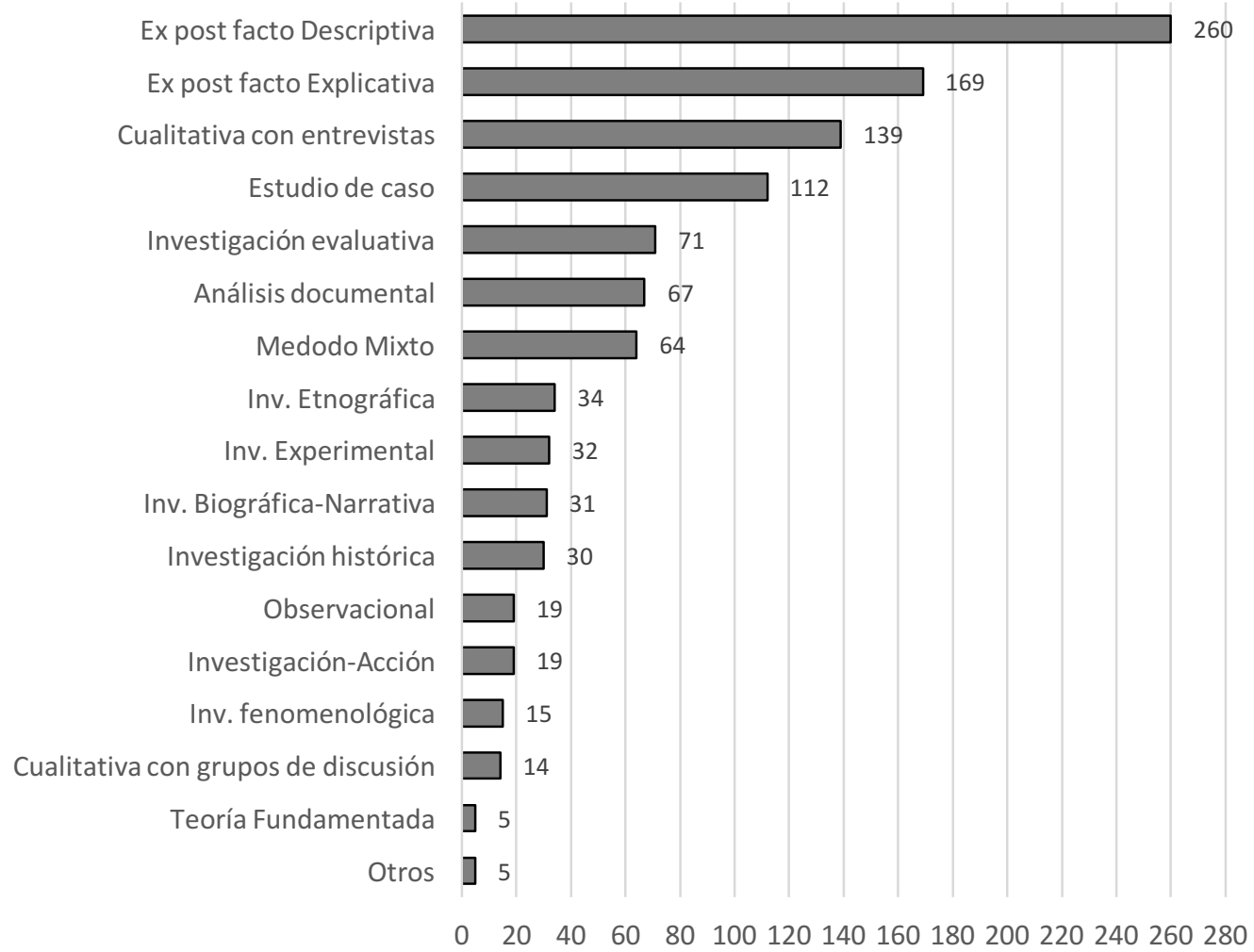

Figura 6. Distribución de los artículos de investigación educativa en América Latina en función del método de investigación utilizado

Fuente: Elaboración propia.

Siendo más concretos en el análisis, se observa que el método más utilizado es el ex post facto de tipo descriptivo, con un $23,9 \%$ del total de las investigaciones, seguido por el método ex post facto de carácter explicativo, con un 15,6\%. A continuación, se encuentran los estudios con entrevistas (12,8\%), los estudios de caso (10,3\%), la investigación evaluativa $(6,5 \%)$, el análisis documental $(6,2 \%)$ y, como se ha comentado, los trabajos que declaran usar un método mixto $(5,9 \%)$. Llama la atención, por otra parte, que en los tres últimos años solo se hayan publicado 19 trabajos de Investigación-Acción en América Latina, que supone un $1,7 \%$ del total.

Una última mirada puede realizarse estudiando los métodos utilizados en los diferentes países de América Latina (cuadro 6). Así destacan algunos países por un enfoque más cuantitativo, especialmente Perú (con el $80 \%$ de sus trabajos), Uruguay y Cuba (ambas 
con el $64,7 \%)$. Otros datos llamativos son el fuerte desarrollo del estudio de caso en Argentina, de la investigación evaluativa y la metodología mixta en Perú y Costa Rica, de los estudios etnográficos en México, de la investigación biográfico narrativa en Brasil y en Colombia, y de la investigación histórica en Brasil.

Cuadro 6. Distribución de artículos de investigación educativa en América Latina en función del método de investigación, por país de los autores

\begin{tabular}{|c|c|c|c|c|c|c|c|c|c|c|c|}
\hline & 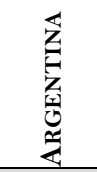 & 恶 & 息 & 通 & 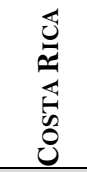 & 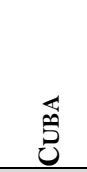 & $\begin{array}{l}0 \\
\circlearrowright \\
\substack{x \\
\Sigma} \\
\sum\end{array}$ & 串 & 恣 & 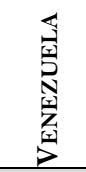 & 嵌 \\
\hline $\begin{array}{l}\text { Ex post facto } \\
\text { Descriptiva }\end{array}$ & 21,05 & 19,54 & 20,59 & 16,44 & 21,15 & 51,61 & 32,21 & 60,00 & 35,29 & 35,00 & 23,94 \\
\hline $\begin{array}{r}\text { Ex post facto } \\
\text { Explicativa }\end{array}$ & 19,30 & 10,91 & 18,14 & 21,92 & 17,31 & 6,45 & 17,79 & 20,00 & 23,53 & 5,00 & 15,19 \\
\hline $\begin{array}{c}\text { Cualitativa con } \\
\text { Entrevistas }\end{array}$ & 15,79 & 15,23 & 13,73 & 12,33 & 9,62 & 3,23 & 9,62 & - & 11,76 & 15,00 & 12,80 \\
\hline Estudio de Caso & 14,04 & 11,17 & 12,25 & 10,96 & 5,77 & 0,00 & 9,62 & - & 5,88 & 10,00 & 10,31 \\
\hline $\begin{array}{c}\text { Investigación } \\
\text { Evaluativa }\end{array}$ & 3,51 & 6,85 & 5,39 & 8,22 & 9,62 & 3,23 & 7,21 & 10,00 & 5,88 & 5,00 & 6,54 \\
\hline Análisis Documental & 3,51 & 9,90 & 3,43 & 4,11 & 9,62 & 16,13 & 0,96 & - & 0,00 & 10,00 & 6,17 \\
\hline Método Mixto & 3,51 & 4,31 & 6,86 & 6,85 & 9,62 & 3,23 & 6,73 & 10,00 & 5,88 & 5,00 & 5,89 \\
\hline Inv. Etnográfica & 3,51 & 2,79 & 1,96 & 4,11 & 1,92 & - & 5,77 & - & 5,88 & ,OO & 3,13 \\
\hline Inv. Experimental & 1,75 & 2,03 & 3,43 & 2,74 & 1,92 & 9,68 & 2,88 & - & 5,88 & 5,00 & 2,95 \\
\hline Biográfica-Narrativa & - & 4,31 & 2,45 & 4,11 & - & - & 2,40 & - & - & - & 2,85 \\
\hline Inv. Histórica & 3,51 & 5,33 & 0,49 & 2,74 & 3,85 & 3,23 & 0,48 & - & - & - & 2,76 \\
\hline Otros & 10,53 & 7,61 & 11,27 & 5,48 & 9,62 & 3,23 & 4,33 & - & - & 10,00 & 7,46 \\
\hline
\end{tabular}

Fuente: Elaboración propia.

\section{Conclusiones}

La presente investigación ofrece una panorámica general de la investigación educativa en América Latina revisando el que tal vez sea el instrumento de comunicación más importante: los artículos publicados en revistas académicas de impacto. Cierto es que no es la única forma de compartir los procesos y resultados de la investigación. Así, existe una importante tradición en la Región en la elaboración de libros, tenemos una extensa cantidad de revistas latinoamericanas no recogidas en las tres indexaciones consideradas, así como revistas de otros lugares del mundo donde los autores y autoras latinoamericanos publican sus investigaciones $\mathrm{y}$, por supuesto, existen estrategias alternativas de comunicación digital, tales como blogs, además de una gran cantidad de informes no publicados. Sin embargo, la exhaustiva revisión de todos los artículos publicados en los tres últimos años, llegando a superar el millar, nos aporta un conocimiento muy aproximado de la situación de la investigación educativa en América Latina.

De entre los muchos resultados encontrados en este trabajo, vamos a destacar algunas ideas especialmente relevantes señalando algunas implicaciones para la mejora de la investigación. Uno de los más importantes hallazgos, no por intuidos menos relevantes, es el desigual desarrollo de la investigación educativa en la Región. Así, tres países concentran el 75\% de los artículos publicados: Brasil, México y Chile. Este hecho sin duda está directamente relacionado con el importante apoyo (al menos en términos relativos) que está recibiendo la investigación educativa en estas dos primeras décadas del siglo XXI 
y que pone sobre la mesa la necesidad de un apoyo institucional para el desarrollo de una investigación educativa de calidad.

En el lado contrario se ha encontrado que la producción académica de algunos países es preocupantemente escasa. Y hablamos no solo de Bolivia, El Salvador, Guatemala, y Paraguay cuyos trabajos no están entre los más de mil artículos analizados, sino también de Panamá, República Dominicana, Puerto Rico, Nicaragua, Honduras o Ecuador. Urge, por tanto, un apoyo institucional decidido para mejorar la investigación educativa de calidad en la Región, muy especialmente en estos países.

No es de extrañar, por otro lado, la íntima relación entre la existencia de revistas académicas indexadas y el número de artículos publicados en cada país. Aquí probablemente se produce un doble efecto: de un lado, la elaboración de buenos trabajos impulsa la existencia de revistas de alta calidad; pero también que se cuente con revistas cercanas hace que publicar sean más sencillo, posiblemente porque ayude a mejorar la calidad de las investigaciones. Esta estrecha relación entre revistas y artículos, sin embargo, no es determinante: el $86 \%$ de los artículos elaborados por investigadores e investigadoras de Argentina se publica en revistas de otros países, cifra que es del $67 \%$ para los autores y autoras colombianos. Ello apunta a la importancia de hacer una fuerte apuesta por la creación de revistas de alta calidad como medio para mejorar la investigación, pero sin descartar la responsabilidad de los investigadores en la misma.

Otro hallazgo, sin duda más sorprendente, es el hecho de que son escasísimos los casos de colaboración entre autores latinoamericanos con otros de fuera de la Región. En esta investigación apenas se han contabilizado un $2 \%$ de los artículos de colaboración "intercontinental". Huelga afirmar que es otra laguna a mejorar en el futuro: la colaboración es un elemento clave para la mejora de la investigación, y la colaboración internacional, si cabe, más aún.

En estas ideas conclusivas sin duda debe reflejarse el papel protagonista encontrado de la mujer en la investigación educativa en América Latina. En Argentina, Cuba y Uruguay, por ejemplificarlo con los datos más extremos, más de un $70 \%$ de los artículos de investigación educativa están firmados por mujeres. Este hecho es coherente con las recientes cifras aportadas por el Instituto de Estadística de la UNESCO que señalan el fuerte liderazgo de las mujeres en la investigación, en general, en América Latina. Efectivamente, mientras que a nivel mundial las mujeres constituyen sólo el 33\% de los investigadores, en la Región latinoamericana el valor promedio es del 46\%. En todo caso, son cifras coherentes con la mayor presencia de mujeres en el mundo de la educación.

Los resultados muestran también que una amplia variedad en las temáticas abordadas por la investigación educativa latinoamericana. Resulta complejo afirmar (o no) que dicha distribución de esfuerzos es la que docentes, directivos y tomadores de decisiones en política educativa pública necesitan. En todo caso es un tópico claro para ser abordado en posteriores indagaciones: ¿los temas responden a las necesidades?

Mucho más llamativos son los resultados respecto a los niveles educativos abordados por las investigaciones. Que una abrumadora cantidad de trabajos sea sobre Educación Superior (el $43,1 \%$ ) es un dato que sorprende y muestra una tendencia a "mirarse el ombligo" por parte de la investigación educativa. Claro que es imprescindible generar conocimiento alrededor de todas las etapas educativas, pero dudamos que lo más importante y prioritario sea la investigación sobre Educación Superior. Y no solo Primaria 
y Secundaria (donde se encuentran la gran mayoría de los estudiantes), también debería ser prioritaria la investigación sobre, por ejemplo, Formación Profesional -una etapa educativa fundamental para el desarrollo social y económico de un país, es abordada solo por el 0,5\% de los artículos- o Educación de Adultos.

Por último, este trabajo ha mostrado que la práctica totalidad de las investigaciones educativas desarrolladas en la Región utilizan bien métodos de enfoque cuantitativo o cualitativo, sin que haya preponderancia de una sobre otra. Esto, de entrada, rompe con el mito de que las revistas de calidad son más reacias a publicar artículos con aproximaciones cualitativas. Está claro que no es cierto. Pero, también, llama la atención los escasos artículos que usan la Investigación-Acción (solo el 1,4\%), enfoques fundamentales para comprender y transformar la realidad educativa. O la mínima producción de las investigaciones autocalificadas "mixtas", menos del 6\% del total. Desde estas líneas, sin embargo, no somos precisamente fervientes defensores de las mismas, por lo que nada diremos sobre ellas.

La investigación educativa, al menos la investigación educativa de calidad, es imprescindible para contribuir a una toma de decisiones informada en todos los niveles educativos: sistema educativo, escuela y aula. Los datos mostrados en el presente estudio permiten la formulación de sugerencias para la mejora destinadas tanto a los centros de investigación y sus investigadores, como a tomadores de decisiones de política pública de los diferentes países de la Región. Efectivamente, el desarrollo de la investigación no puede ser solo iniciativa de los investigadores. Parafraseando a Charles de Gaulle "La investigación es demasiado seria para dejarla en manos de investigadores". Las administraciones han de tener un papel activo en el fomento de la investigación, a través de diferentes estrategias. Entre ellas, es posible señalar tres:

- Apoyo decidido a la investigación y la profesión investigadora. Es necesario mejorar las condiciones económicas y laborales, los apoyos y los reconocimientos de los y las investigadores. Según los últimos datos del Banco Mundial sobre el gasto en I+D de 2015 , el porcentaje del PIB promedio destinado a la investigación en América Latina es la décima parte $(0,24 \%$ del PIB) de lo que destinan otras regiones como la Unión Europea (2,05\%) o América del Norte (2,79\%). No tenemos datos de la inversión en investigación educativa, pero a la vista de los productos, la necesidad de un apoyo decidido es más que necesario.

- Una clara apuesta por la carrera investigadora. La formación en investigación educativa es aún un reto pendiente en las universidades que requiere de especial atención. No estaría muy alejado de la realidad afirmar que una gran parte de la falta de calidad de los trabajos desarrollados en América Latina se debe al uso deficiente de la metodología. Sin una buena formación, sin materias de investigación en posgrados y un refuerzo de los doctorados en educación, difícilmente mejorará la investigación.

- Apoyos a la difusión del conocimiento. Es decir, fomentar la creación de revistas de calidad y, especialmente, favorecer el desarrollo y mejora de las existentes. Para ello, es prioritario generar sistemas de garantía de calidad de los procesos editoriales y de evaluación de los artículos, o profesionalizar la labor de la edición, sin olvidar la apuesta por el acceso libre, entre otros. 
Por último, fomentar las relaciones entre grupos de investigación educativa, la creación de redes y la interdisciplinariedad puede ser un apoyo para la mejora de la investigación educativa. Experiencias como el Consejo Mexicano de Investigación Educativa (COMIE), la Asociación Chilena de Investigadores en Educación (ACHIE), la Sociedad de Investigación Educativa Peruana (SIEP) o la recién creada Asociación Ecuatoriana de Fomento de la Investigación Educativa (ASEFIE), por poner algunos ejemplos, son una excelente estrategia.

¿Es lo mismo investigar y publicar? No exactamente, pero no son actividades tan lejanas. Tal y como señalan Murillo, Martínez-Garrido y Belavi (2017, p. 6): "En investigación, lo que no está escrito no existe" por lo que no se puede hablar de investigación si no se traduce en artículos. Y un artículo de investigación ha de ser necesariamente producto de un proceso de indagación empírica. Por eso, esta investigación ha buscado describir el estado actual de la investigación educativa en América Latina a partir de los artículos de investigación empírica. Entre sus limitaciones está no analizar los artículos publicados en revistas editadas en otros países del mundo, en ocasiones más exigentes que las de América Latina, pero llegar a ellos supone un trabajo más que imposible. Sin embargo, la revisión de más del millar de artículos ha aportado una visión panorámica de suficiente detalle que da pie a la realización de futuros trabajos sobre estos artículos publicados en "otras revistas", o estudios que relacionen la producción científica con el número de académicos en cada país. el análisis sobre temas concretos de estudio, métodos de investigación o países.

Sin investigación educativa de calidad, desarrollada en los contextos de análogas características, resulta imposible mejorar la calidad de la educación. Así, en América Latina, región que concentra quizá a algunos de los países con mayores desafíos, es fundamental que se refuerce el desarrollo de una investigación educativa propia, que recoja las problemáticas de sus escuelas, identifique sus propias estrategias para la mejora y promueva el desarrollo de políticas educativas contextualizadas a la realidad de sus aulas. Apostamos firmemente por una investigación educativa de calidad.

\section{Referencias}

Abraham, M. y Rojas A. (1997). La investigación educativa latinoamericana en los últimos diez años. Revista de Educación, 312, 21-42.

Arredondo, M., Martínez, S., Mingo, A. y Wuest, T. (1984). La investigación educativa en México. Un campo científico en proceso de constitución. Revista Mexicana de Sociología, 46(1), 5-38. https://doi.org/10.2307/3540290

Centro de Investigación y Experimentación Pedagógica. (1982). Inventario de instituciones y metodologías de discriminación de investigaciones educativas que están en práctica en América Latina y el Caribe. Montevideo: CIEP.

Cisternas, T. (2011). La investigación sobre formación docente en Chile: Territorios explorados e inexplorados. Calidad en la Educación, 35, 131-164. https://doi.org/10.4067/s071845652011000200005

Colina Escalante, A. (2011). El crecimiento del campo de la investigación educativa en México. Un análisis a través de sus agentes. Perfiles Educativos, 33(132), 8-26. https://doi.org/10.22201/iisue.24486167e.2011.132.24894 
COMIE. (2004). La investigación educativa en México: Usos y coordinación. Revista Mexicana de Investigación Educativa, 8(19), 847-898.

De Landsheere, G. (1985). History of educational research. International encyclopedia of education. En T. Husen y T. N. Postlethwaite (Eds.), The international encyclopedia of education. Research and studies (pp. 1588-1596). Nueva York, NY: Pergamon Press.

Flores-Crespo, P. (2009). Investigación educativa y políticas públicas en México: Una relación amorfa y elusiva. Sinéctica, 33, 1-13.

García Huidobro, J. E. y Ochoa, J. (1978). Tendencias de la investigación en educación en América Latina. Santiago de Chile: CIDE.

Gorostiaga, J. M., Nieto, G. y Cueli, F. (2014). Un acercamiento al campo argentino de producción de conocimiento educativo a través de las publicaciones en revistas académicas durante 2001-2010. Revista de la Escuela de Ciencias de la Educación, 9, 247-270.

Gorostiaga, J. M., Palamidessi, M. I. y Suasnábar, C. (2016). Revistas académicas e investigación educativa en Argentina: Situación actual y perspectivas futuras. Propuesta Educativa, 45, 91106.

Gutiérrez Serrano, N. G. (1998). Orígenes de la institucionalización de la investigación educativa en México. Revista Mexicana de Investigación Educativa, 3(5), 13-38.

Hanushek, E. A. (1997). Assessing the effects of school resources on student performance: An update. Educational Evaluation and Policy Analysis, 19(2), 141-164. https://doi.org/10.3102/01623737019002141

Latapí, P. (2008). ¿Recuperar la esperanza? La investigación educativa entre pasado y futuro. Revista Mexicana de Investigación Educativa, 13(36), 283-297.

Miguel, S. (2011). Revistas y producción científica de América Latina y el Caribe: Su visibilidad en SciELO, RedALyC y Scopus. Revista Interamericana de Bibliotecología, 34(2), 187-199.

Murillo, F. J. y Román, M. (2011). School infrastructure and resources do matter: Analysis of the incidence of school resources on the performance of Latin American students. School Effectiveness and School Improvement, 22(1), 29-50. https://doi.org/10.1080/09243453.2010.543538.

Murillo, F. J., Martínez-Garrido, C. y Belavi, G. (2017). Sugerencias para escribir un buen artículo científico en educación. REICE. Revista Iberoamericana sobre Calidad, Eficacia y Cambio en Educación, 15(3), 5-34. https://doi.org/10.15366/reice2017.15.3.001

Packer, A. L., Cop, N., Luccisano, A., Ramalho, A. y Spinak, E. (2014). SciELO, 15 Años de acceso abierto: Un estudio analítico sobre acceso abierto y comunicación científica. París: UNESCO. https://doi.org/10.7476/9789233012370

Palamidessi, M. I., Gorostiaga, J. M. y Suasnábar, C. (2014). El desarrollo de la investigación educativa y sus vinculaciones con el gobierno de la educación en América Latina. Perfiles Educativos, 36(143), 49-66. https://doi.org/10.1016/So 185-2698(14)70609-9

Rivero, J. (1994). Investigación educativa en América Latina: La agenda pendiente. Políticas y estrategias prioritarias, actores, temas. Proyecto Principal de Educación en América Latina y el Caribe, 34, 83-108.

Shiefelbein, E. (1990). La investigación educativa en América Latina: De la fase artesanal a la fase industrial. Perspectivas, 20(1), 61-67.

Soto Roa, F. (2012). Veinte años de investigación en educación escolar en Chile 1990-2010. Santiago de Chile: Ministerio de Educación.

UNESCO. (2015). Informe de la UNESCO sobre la Ciencia: Hacia 2030. París: UNESCO. 
Villalobos, C., Band, A., Torres, M. y González, S. (2016). Lógicas y modos de producción de conocimiento en política educativa: Análisis de la investigación producida en Chile (20002011). Revista Iberoamericana de Ciencia Tecnología y Sociedad, 11(33), 9-32.

\section{Breve CV de los autores}

\section{F. Javier Murillo}

Director de la Cátedra UNESCO en Educación para la Justicia Social de la Universidad Autónoma de Madrid. Profesor titular en Métodos de Investigación y Evaluación en Educación de la UAM. Coordinador del grupo de investigación "Cambio Educativo para la Justicia Social" y Secretario Académico del Instituto de Derechos Humanos, Democracia, y Cultura de Paz y No Violencia (DEMOSPAZ) de la UAM. Fue Coordinador General del Laboratorio Latinoamericano de Evaluación de la Calidad de la Educación (LLECE), de la UNESCO, y Director de Estudios del Centro de Investigación y Documentación Educativa (CIDE) del Ministerio de Educación de España. Es Coordinador de la Red Iberoamericana de Investigación sobre Cambio y Eficacia Escolar (RINACE), Director de REICE. Revista Iberoamericana sobre Calidad, Eficacia y Cambio en Educación, de la Revista Iberoamericana de Evaluación Educativa y de Revista Internacional de Educación para la Justicia Social. Página web: https://www.fjaviermurillo.es. ORCID ID: https://orcid.org/O000-0002-8003-4133. Email: javier.murillo@uam.es

\section{Cynthia Martínez-Garrido}

Profesora Ayudante Doctor en Métodos de Investigación y Diagnóstico en Educación en la Universidad Autónoma de Madrid. Premio Extraordinario de Doctorado por la UAM. Premio a la mejor Tesis Doctoral 2015 por la Asociación Interuniversitaria de Investigación Pedagógica (AIDIPE). Miembro del grupo de investigación "Cambio Educativo para la Justicia Social" (GICE) de la UAM. Ha realizado su postdoctorado en la Universidad de Durham (Reino Unido) y la Universidad de Granada (España) bajo el contrato Juan de la Cierva del Ministerio español de Ciencia, Innovación y Universidades. Además, ha realizado estancias de investigación en la UCLA-Universidad de California (Los Ángeles, EEUU) y en la Universidad de Uppsala (Suecia). Es Editora de REICE. Revista Iberoamericana sobre Calidad, Eficacia y Cambio en Educación y de la Revista Latinoamericana de Educación Inclusiva. Sus líneas de investigación son Enseñanza eficaz, Segregación escolar, Liderazgo educativo y Educación para la Justicia Social. Página web: www.cmartinezgarrido.es. ORCID ID: https://orcid.org/oooo-0001-7586o628. Email: cynthia.martinez@uam.es 